This is the post-print (ie final draft post-refereeing) version of the following article Davis AM, Perruccio AV, Ibrahim S, Hogg-Johnson S, Wong R, Badley EM.

Understanding recovery: Changes in the relationships of the International Classification of Functioning (ICF) components over time. Soc Sci Med. 2012 Dec;75(11):1999-2006, which has been published in the final form at: http://www.ncbi.nlm.nih.gov/pubmed/22940011

\title{
Understanding recovery: Changes in the relationships of the International Classification of Functioning (ICF) components over time
}

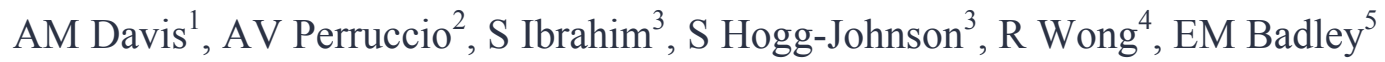 \\ on behalf of the ICF Research Team
}

1. Division of Health Care and Outcomes Research, Toronto Western Research Institute;

Departments of Rehabilitation Science and Institute of Health Policy, Management and

Evaluation, University of Toronto, Toronto, Canada

2. Department of Orthopaedic Surgery, Toronto Western Hospital and Institute of Health Policy, Management and Evaluation, University of Toronto, Toronto, Canada

3. Institute for Work and Health, Toronto, Canada

4. Division of Health Care and Outcomes Research and Arthritis Community Research and Evaluation Unit, Toronto Western Research Institute, Toronto, Canada;

5. Division of Health Care and Outcomes Research, Toronto Western Research Institute and Dalla Lana School of Public Health, University of Toronto, Toronto, Canada

Corresponding author: Aileen M. Davis, $\mathrm{PhD}$

MP11-322, 399 Bathurst Street

Toronto, ON, Canada M5T 2S8

Tel: 416 603-5543 
Fax: 416 603-6288

Email: adavis@uhnresearch.ca

Key words: International Classification of Functioning, Disability and Health; longitudinal data; path analysis, disability, intervention 


\begin{abstract}
The International Classification of Functioning, Disability and Health framework describes human functioning through body structure and function, activity and participation in the context of a person's social and physical environment. This work tested the temporal relationships of these components. Our hypotheses were: 1) there would be associations among physical impairment, activity limitations and participation restrictions within time; 2) prior status of a component would be associated with future status; 3 ) prior status of one component would influence status of a second component (e.g. prior activity limitations would be associated with current participation restrictions); and, 4) the magnitude of the within time relationships of the components would vary over time. Participants $(n=931)$ with primary hip or knee joint replacement, an intervention with predictable improvement in pain and disability, completed standardized outcome measures pre-surgery and five times in the first year post-surgery. These included physical impairment (pain), activity limitations and participation restrictions. ICF component relationships were evaluated cross-sectionally and longitudinally using path analysis adjusting for age, sex, BMI, hip vs. knee, low back pain and mood. All component scores improved significantly over time. The path coefficients supported the hypotheses in that both within and across time, physical impairment was associated with activity limitation and activity limitation was associated with participation restriction; prior status and change in a component were associated with current status in another component; and, the magnitude of the path coefficients varied over time with stronger associations among components to three months post surgery than later in recovery with the exception of the association between impairment and participation restrictions which was of similar magnitude at all times. This work enhances
\end{abstract}


understanding of the complexities of the ICF component relationships in evaluating disability over time. Further longitudinal studies including evaluation of contextual factors are required.

\section{Introduction}

The understanding of health, disease and their consequences has been underpinned by advances in our theoretical understanding of disability and the disablement process (Masala \& Petretto, 2008). While a number of theoretical models have been developed, the World Health Organization's International Classification of Functioning, Disability and Health (ICF) (World Health Organization, 2001) is a framework that has been widely adopted to support health and disability reporting and evaluation across a multitude of health conditions in a variety of care settings (Masala \& Petretto, 2008; Ustun et al., 2003; World Health Organization, 2001). It is widely used as the supporting framework for choosing and guiding the content development of outcome measures (Canadian Institutes of Health Information., June 2001.; Hamilton et al., 1987; Pisoni et al., 2008; Riddle et al., 2008; Schwarzkopf et al., 2008; Xie et al., 2008).

The ICF is an integrative model for describing human functioning and the impact of health (World Health Organization, 2001) with health conditions described as conditions, diseases, disorders, injuries, etc. and functioning captured through the components of body structure and function, activity and participation in the context of a person's social and physical environment. Disability is an overarching term for impairments in body structure or function, activity limitations and participation restrictions. "Impairment are problems in body function or structure such as a significant deviation or loss" (World Health Organization, 2001, Page 12) "Activity limitations are difficulties an individual may have in executing activities" (World Health Organization, 2001, Page 14) and may range from a slight to severe deviation in terms of quality or quantity compared to that expected of people without the health condition. 
"Participation restrictions are problems an individual may experience in involvement in life situations" (World Health Organization, 2001, Page 14). Personal or environmental contextual factors may facilitate or hinder performance across ICF components. Despite defining activity limitations and participation restrictions separately and diagrammatically showing these components separately (World Health Organization, 2001, Page 18), coding of items within the ICF combines activity and participation (World Health Organization, 2001) leading to conceptual confusion and debate. A number of authors argue that activity and participation are distinct and should not be combined (Badley, 2008; A. M. Jette et al., 2003; Nordenfelt, 2003; Schuntermann, 2005; Simeonsson et al., 2003) and still others have demonstrated that activity and participation are two distinct constructs (A. M. Jette et al., 2007; Pollard et al., 2010). In this study, we also considered activity limitations and participations restrictions as separate components not only because we too would argue that they are distinct but because, as we describe below, out clinical knowledge of the recovery of people with hip or knee replacement (TJR) who represent our study sample supports activity limitation and participation restrictions as separate components.

Despite the adoption of the ICF framework, there has been little testing of the relationships among the components. Some authors have evaluated the component relationships using cross-sectional data. Rusch et al. found that impairments of body function and activity limitations were associated with restrictions in social role participation in a cross-sectional study of people with HIV/AIDs (Rusch et al., 2004). Slim et al. found that impairments of the hand and feet were associated with activity limitations, and that impairment of the hands was associated with participation restrictions in people with leprosy (Slim et al., 2010). In the context of arthritis, others also have identified relationships among impairment, activity limitation and 
participation restriction (Dagfinrud et al., 2005; Dekker et al., 2010; Pollard et al., 2010). Still others have evaluated predictors of participation restrictions and found a mediated model of physical symptoms (impairments), activity limitations and depression (impairment) in people with arthritis (Machado et al., 2008). Additionally, in people with distal radius fracture, although impairments, activity limitations and participation restrictions were associated with health status, activity limitations were the dominant predictor of health status (J. E. Harris et al., 2005). While these data provide some insight into the relationships of the ICF components, there is still a need to understand the component relationships over time and in the context of intervention and recovery.

The application of the ICF framework has not considered time and many cross-sectional studies include participants at different times in the disease course or time of recovery. There is an urgent need to demonstrate whether the cross-sectional relationships among components changes over time during recovery and to understand the longitudinal relationships of the components. Testing this assumption of lack of variability of the component associations is a critical first step that will enable more comprehensive evaluation of the ICF framework including understanding how personal and environmental factors affect impairment, activity limitation and participation restriction and the component relationships both cross-sectionally and longitudinally.

We address these knowledge gaps and evaluated the relationships of the ICF components both within and across time in people undergoing primary total hip (THR) or total knee replacement (TKR) for osteoarthritis (OA). Our objectives were: 1) to evaluate the relationships of the components of the ICF cross-sectionally; 2) to determine if the relationships of the 
components are variable over time; and, 3) to evaluate longitudinally, the relationships of the components of the ICF.

TJR for OA is a well established, effective procedure (Bellamy et al., 1988; Chang et al., 1996; Fortin et al., 1999; Fortin et al., 2002; W. H. Harris \& Sledge, 1990; Judge et al., 2010; Laupacis et al., 1993) in which we could confidently predict changes in the ICF components over time. The pre-surgery evaluation provides a defined baseline which is often challenging in chronic diseases as a true onset of the disease can be difficult to define. Additionally, with low morbidity and mortality (Institute for Clinical Evaluative Sciences., 2000), we can confidently predict a rapid improvement that takes place over a feasible time-period for study (i.e. one year).

Based on clinical knowledge of people with TJR, in the weeks immediately following surgery, we expect the biggest change to occur in impairment of body structure and function (e.g. manifested by pain) with fewer changes in activity limitations and participation restrictions. Over time, impairment may plateau and greater change in activity and, eventually, participation might occur. As a consequence of the different rates of change in the components, the relationships among components are expected to change over time. Given this clinical scenario and the predictability of the effectiveness of the intervention, TJR patients provides an ideal group for study and positioned us to test the following hypotheses: 1) there would be associations among physical impairment, activity limitations and participation restrictions within time; 2) prior status of a component would be associated with future status; 3) prior status of one component would influence current status of a second component (e.g. prior activity limitations would be associated with current participation restrictions etc.); and, 4) the magnitude of the within time relationships of the components would vary over time.

\section{Methods}




\section{Study design and setting}

This prospective longitudinal study recruited participants 18 years and over from four tertiary care centers in Toronto, Canada who were undergoing primary TJR surgery and subsequent rehabilitation based on a standardized care pathway. Participants had sufficient English fluency to complete the questionnaires. Informed consent was obtained in accordance with the approval of the participating institutions' ethics review boards.

The 931 participants (437 with THR and 494 TKR) completed baseline questionnaires within two weeks prior to surgery and at 2 weeks, 1, 3, 6 and 12 months post-surgery. In addition to the standardized outcome measures representing physical impairments, activity limitations and participation restrictions described below, age, sex, education level, body mass index (BMI), hip or knee replacement, comorbidity, and mood were recorded pre surgery. Comorbidity was recorded based on a no/yes response to the listing of conditions (Sangha et al., 2003). Mood was measured by the Hospital Anxiety and Depression Scale (HADS) (Zigmond \& Snaith, 1983) and the fatigue subscale of the Profile of Mood State (POMS) (O'Connor, 2004). The HADS has been widely used in community-based and outpatient populations (Herrmann, 1997). The anxiety and depression subscales consist of 7 items scored 0-3 with a summed score ranging from 0-21 where higher scores indicate more anxiety and depression. The POMS is a frequently used measure of fatigue and has been used in studies of a range of chronic conditions (O'Connor, 2004). Fatigue was evaluated by 5 items scored 0-'not at all' to 4-'extremely'; scores are summed. A single variable representing mood was constructed by converting each of the three scores to 0 to 10 and summing them to create a mood score (Cronbach's alpha 0.77) ranging from 0 to 30 where zero represented no problems in mood. 


\section{Standardized Measures representing ICF Components}

Standardized outcome measures commonly used in TJR were chosen to represent the ICF components wherever possible. For some components, more than one measure was included to ensure we 'covered' relevant content and also to address some criticisms of the standardized measures. For example, the Western Ontario and McMaster Universities' Osteoarthritis Index (WOMAC) (Bellamy et al., 1988) pain items which are part of the Hip Disability (HOOS-pain) (Nilsdotter et al., 2003) and Knee Injury and Osteoarthritis Outcome Score (KOOS-pain) (Roos et al., 1998) reflect pain on activity. Hence, we also included the Chronic Pain Grade (Von Korff et al., 2000; Von Korff et al., 1992) as a measure of pain intensity. Similarly, the WOMAC function items do no cover the spectrum of difficulty (i.e. more difficult activities are not included) (Davis et al., 2003; Ryser et al., 1999), hence, we included the higher demand HOOS/KOOS function in sport and recreation subscale, e.g. squatting etc. (Nilsdotter et al., 2003; Roos et al., 1998).

\section{Impairment of Body Structure or Function}

Physical impairments as manifested by pain were measured using the HOOS-pain (Nilsdotter et al., 2003) and KOOS-pain (Roos et al., 1998) subscales for THR and TKR respectively and the Chronic Pain Grade (Von Korff et al., 2000; Von Korff et al., 1992). HOOSand KOOS-pain subscales assess the extent of pain during activities such as 'walking on a flat surface' and 'going up and down stairs'. The HOOS-pain and KOOS-pain are 10- and 9-item scales respectively with response options ranging from 0-'none' to 4-'extreme' and scores are summed. The Chronic Pain Grade measures pain intensity based on the sum of the responses to 
three questions with scores ranging from 1-'no pain' to 10-'pain as bad as it could be' and the scores are summed.

\section{Activity Limitations}

Activity limitations were measured with the WOMAC Likert version 3.0 function subscale (Bellamy et al., 1988) and the HOOS/KOOS Function in sport and recreation subscale (Nilsdotter et al., 2003; Roos et al., 1998). The WOMAC evaluates basic mobility and activities

of daily living (walking on flat ground, rising from sitting, climbing stairs etc.) in 17 questions with response options ranging from 0-'not at all difficult' to 4-'extreme difficulty'; scores are summed. Using identical response options, the HOOS/KOOS subscales for THR and TKR respectively evaluate more demanding activities than those in the WOMAC in 4 and 5 items respectively. Scores are summed and converted to a 0-100 score.

\section{Participation Restrictions}

We used the Late Life Disability Instrument (LLDI) (A. M. Jette et al., 2002) in which respondents separately rate the extent and frequency with which they feel limited in their ability to personally perform 16 socially expected life tasks (e.g. participating in active recreation, visiting friends and family, volunteering etc.) on a 1-'completely' to 5-'not at all' scale to measure participation. Raw summed scores are converted to scaled interval level summary scores ranging from 0-100 where higher scores represent higher frequency and less limitations respectively (A.M. Jette et al., 2006). Additionally, respondents completed the Calderdale Rheumatic Disablement Survey community mobility measure (Badley \& Tennant, 1993) that assessed the extent to which a respondent's chronic condition limits their mobility or ability to travel within their community on a 4 items scored 1-'none' to 5-'can no longer do'; scores are summed. 
For ease of comparison, after scoring each measure according to published instructions, each measure score was converted to a 0-10 scale. Where necessary, measures were reversed scored so that higher scores indicated worse health/more difficulty. Measured variables were then constructed by summing the individual measure scores within the ICF component after evaluating Cronbach's alpha for the individual measures (data not shown) and the ICF components. Internal consistency for physical impairments over time ranged from 0.83 to 0.88 , from 0.68 to 0.71 (with the exception of 0.58 at 2 weeks post-surgery) for activity limitations from 0.77 to 0.84 (except for 0.69 at 2 weeks) for participations restrictions. Additionally, unidimensionality of the components was demonstrated through bi-factor analysis (Lai et al., 2006). Little et al. suggest that such parceling of items to a measure or individual measures to a single score reduces measurement error and is parsimonious limiting the number of parameters estimated in modeling (Little et al., 2002). The final measured variable ranged from 0-20 for each of impairments and activity limitations and from 0-30 for participation restrictions.

\section{Analysis}

Descriptive statistics and measures of association were conducted for all variables using the Statistical Analysis System version 9.0 software (SAS Institute Inc., 2005). Data were checked for outliers and for normality using the skewness and kurtosis values (Kline, 2005) and no data transformation was required.

Path analysis using Mplus 5.21 (Muthen \& Muthen) was used to evaluate the relationships of the ICF components within and across time. Our a priori model (Figure 1) reflects our hypotheses where we expected: each of the ICF components to be associated within each time point (vertical pathways) such that there would be direct pathways from impairment to 
activity limitations, activity limitations to participation restrictions and from impairment to participation restrictions (hypothesis 1); direct pathways across time within each of the components, i.e. impairment to impairment across time, activity limitations to activity limitations across time and participation restrictions to participation restrictions across time (horizontal pathways) (hypothesis 2); and, direct relationships among components where prior status of one component would be associated with another component at a subsequent time e.g. impairment at time 0 would be associated with activity limitations at time 1 which would be associated with participation restrictions at time 2 etc. (diagonal pathways) (hypothesis 3). Additionally, Figure 1 shows indirect pathways from impairment to participation through activity limitation; e.g. from impairment at time 0 to participation restrictions at time 1 through activity limitations at time 1 etc. and from impairment at time 0 to participation restrictions at time 2 through activity limitations at time 1 etc.

We first developed our base model as described below to evaluate hypotheses 1,2 and 3 . As suggested in the literature (Bollen \& Long, 1993), overall model fit was examined with several indices including chi-square test (although with large sample size the chi-square test is often significant), Root Mean Square Error of Approximation (RMSEA), Comparative fit Index (CFI), Tucker-Lewis Index (TLI) and Standardized Root Mean Square Residual (SRMR). Good fit was supported by: RMSEA $\leq 0.05$ with a $90 \%$ upper confidence limit $<0.08$ and nonsignificant p-value; $\mathrm{SRMR} \leq 0.08$; and, CFI and TLI $\geq 0.95$ (Browne \& Cuceck, 1993; Hu \& Bentler, 1998). As described in the results, the hypothesized model did not display good fit. However, after modification based on plausible relationships (Bachmeier et al., 2001; MacCallum et al., 1992) and adjustment for covariates (age, sex, hip versus knee replacement, BMI, low back pain and mood) that have demonstrated relationships to the outcomes used to 
represent impairment, activity limitations and participation restrictions (Bachmeier et al., 2001; Bourne et al., 2007; Jones et al., 2007; Jones et al., 2000; Krushell \& Fingeroth, 2007; Naylor et al., 2008), we achieved good fit. This model then became our base model against which we tested nested models using the chi-square difference test. Specifically, against this base model, we tested paths with equality constraints where the component relationships within time were constrained to be equal at all time points (i.e. impairment to activity limitations at time zero were constrained to be equal to impairment to activity limitations at time one etc.) (hypothesis 4). Where a significant difference was detected, this was interpreted as a variable or time-dependent relationship over time, and equality constraints were not retained. However, where no statistically significant differences were found between nested models, the respective relationships were interpreted to represent lack of variability in the respective relationships over time and the equality constraints were retained in the model. Finally, we tested models where paths from previous impairment to current activity limitations were constrained to be equal (i.e. the coefficients for impairment at time 0 to activity limitations at time 1 would be equal to impairment at time 1 to activity limitations at time 2 etc.) and where paths from previous activity limitations to current participation restrictions were constrained to be equal (i.e. the coefficients from activity limitations at time 0 to participation restrictions at time 1 would be equal to activity limitations at time 1 to participation restrictions at time 2 etc.).

The final model that is described later was estimated using Maximum Likelihood with a mean adjusted chi-square test statistic to take account of any small deviations from normality (Finney \& DiStefano, 2006). The direct maximum likelihood (DML) estimation method (Enders, 2010) was used to handle missing data, assuming data were 'missing at random'. Parameter estimates generated using DML are consistent and efficient (Allison, 2003). Overall, 85\% of 
participants had complete data for the outcome measures at all time points.

\section{Results}

The mean age of the sample $(\mathrm{n}=931)$ was 63 years (range $31-88)$ and $60 \%$ were female. Forty-six percent had more than high school education and $34 \%$ of the sample continued to work either part-time or full-time. Forty percent were obese (i.e. BMI $>30$ ). Hypertension (44\%), low back pain (22\%), diabetes $(12 \%)$ and respiratory disease $(9 \%)$ were common.

As anticipated, the sample had severe physical impairments, activity limitations and participation restrictions prior to surgery (Table 1). Large improvements occurred through 3 months post TJR for all ICF components followed by smaller improvement through six and twelve months post surgery.

The hypothesized model (Figure 1) did not display overall good fit; chi-square=1457 (degrees of freedom=200), $\mathrm{CFI}=0.895, \mathrm{TLI}=0.863, \mathrm{RMSEA}=0.086$, and $\mathrm{SRMR}=0.129$. Fit improved with adjustment for covariates (chi-square=1160 (degrees of freedom=196), $\mathrm{CFI}=0.920, \mathrm{TLI}=0.893, \mathrm{RMSEA}=0.076, \mathrm{SRMR}=0.102)$ and, subsequently including longer-term effects (of activity limitations at 3 months to 12 months post surgery and from pre-surgery participation restrictions to each post-operative time) resulted in a model that displayed good fit (chi-square $=781.3$ (degrees of freedom $=186$ ), $\mathrm{CFI}=0.950, \mathrm{TLI}=0.930, \mathrm{RMSEA}=0.061$, $\mathrm{SRMR}=0.084)$. The chi-square difference test $(\mathrm{p}<0.0001)$ confirmed significant improvement with this model over the hypothesized model. Hence, this modified model with adjustment for covariates and the additional pathways became our base model and supported hypotheses 1,2 and 3 described subsequently in the description of the final model (Figure 2). The exception was 
at 2 weeks post surgery where there was no significant relationship between impairment and participation restrictions as per hypothesis 1 .

Sequential testing of models (hypothesis 4) where paths from current values of physical impairment to activity limitations, activity limitations to participation restrictions and impairment to participation restrictions were constrained to be equal at each time point versus the base model resulted in statistically significant chi-square difference tests demonstrating that the cross-sectional relationships vary over time (physical impairment to activity limitations: $\Delta \mathrm{x}^{2}(\mathrm{df})=15.6(5), \mathrm{p}=0.0081$; activity limitations to participation restrictions: $\Delta \mathrm{x}^{2}(\mathrm{df})=123.3(5)$, $\mathrm{p}<0.0001$; and, impairment to participation restrictions: $\left.\Delta \mathrm{x}^{2}(\mathrm{df})=24.8(5), \mathrm{p}=0.0002\right)$.

In attempting to achieve a parsimonious final model, we compared sequential models where we constrained the paths from prior physical impairments to current activity limitations and then prior activity limitations to current participation restrictions to be equal to the base model. The chi-square difference test was not statistically significant for either of these nested models (prior physical impairments to current activity limitations: $\Delta x^{2}(d f)=3.6(4), p=0.4628$; and, prior activity limitations to current participation restrictions: $\left.\Delta x^{2}(d f)=5(4), p=0.2873\right)$ and these constraints were contained in our final model. As shown by the unstandardized coefficients in table 2, the constrained coefficient for prior impairment to current activity limitations was 0.212 and -0.297 for prior activity limitation to current participation restriction.

Our final model had good fit (chi-square 731.7(194), CFI=0.949, TLI=0.932, RMSEA $=0.057, \mathrm{SRMR}=0.084$ ). Figure 2 shows the final adjusted model based on standardized coefficients. This model demonstrates, supporting hypothesis 1 (and hypothesis 4 given that there were significant differences in testing the constraints as noted above) that within time impairments are related to activity limitations (coefficients range from 0.661 to 0.337 with 
stronger relationships through three months post surgery); activity limitations are associated with participation restrictions with coefficients ranging from 0.458 and stronger to 3 months post surgery and 0.399 and 0.335 at 6 and 12 months post surgery respectively; and, impairment significantly associated with participation restrictions at all time points but 2 weeks post surgery, although the coefficients were modest ranging from 0.10 to 0.20 .

Similarly, the final model supports hypothesis 2 as prior status influences future status within component, physical impairment, activity limitation and participation restrictions. The standardized coefficients across time for physical impairments were large from 2 weeks to 12 months post surgery (range 0.699 to 0.730 ) demonstrating the strong relationship between prior status and current status. The relationship was much weaker between pre-surgery and 2 weeks post-surgery (0.258). Similarly for activity limitations prior status was associated with current status with standardized coefficients ranging from 0.403 to 0.712 . Again between pre-surgery and 2 weeks post surgery, the relationship was weakest with a coefficient of 0.403 . For participation restrictions coefficients ranged from 0.242 to 0.741 with the low coefficient between pre-surgery and 2 weeks post surgery. In the context of joint replacement as an intervention, we interpret the pattern of coefficients within a component to indicate that physical impairments and activity limitations improve rapidly and then stabilize, and participation restrictions continue to improve over time although less so as time passes.

There were significant pathways from prior impairment to current activity limitations and from prior activity limitations to current participation restrictions (hypothesis 3). In each case, the coefficients on the paths were negative. These negative coefficients on the diagonals as shown in Figure 2 represent the effect of the change that occurs over time in one component on 
the status of another component such that the larger the improvement in, for example, physical impairments between times 1 and 2 the less activity limitations at time 2 and so forth.

We also note that in addition to the pathways we hypothesized, in order to achieve a good fitting base model against which to test nested models for our a priori hypotheses, in addition to adjustment for covariates, additional paths that were not hypothesized were identified.

Specifically, in the longer term, prior activity limitations (at 3 months) influenced future activity limitations (at 12 months); and, participation restrictions prior to surgery influenced participation restrictions at all subsequent time points.

\section{Discussion}

Despite the introduction of the ICF framework for disability and health in 2001 (World Health Organization, 2001), there has been little evaluation of the relationships of impairment, activity limitation and participation restrictions, and none to our knowledge simultaneously evaluating the relationships of the components longitudinally. This work applied the ICF framework, specifically impairment of body function and structure, activity limitations and participation as three components, in a clinical context in which people were recovering following TJR.

Our work has clinical implications for use and interpretation of patient outcomes given the variability in the component relationships over time. Specifically, cross-sectional studies need to include samples that are at a similar point of recovery and longitudinal evaluation needs to consider the change in related outcomes. We found that prior status within a component affected later status such that less impairment was associated with less impairment at subsequent times and so forth for activity limitations and participation restrictions. There were lagged 
relationships as well, as activity limitations at 3 months were associated with activity limitations at 12 months and participations restrictions prior to surgery were associated with participation restrictions at all subsequent follow-ups. Importantly, change in one component was associated with current status of another component, i.e. greater change in physical impairments was associated with less activity limitations and greater change in activity limitations was associated with less participation restrictions. In the application of the ICF framework in clinical contexts and interventions, these findings suggest that it is critical to consider the time of evaluation of the component as well as the simultaneous effect of one component (or change in one component) on other components. The implications of these findings for outcome measurement are significant for looking at change in outcome. Change needs to be interpreted in the context of more proximal time points of the ICF components. Additionally, studies evaluating change in ICF components in isolation need to be interpreted with caution if inferences are made about the consequences of this change for other components.

Similar to our work, others have found that impairments were associated with activity limitations and participation restrictions and activity limitations were associated with participation restrictions within time (Dagfinrud et al., 2005; Dekker et al., 2010; Rusch et al., 2004; Slim et al., 2010). None of these authors conducted their work in individuals with people with osteoarthritis, or more specifically TJR. Other TJR studies largely have focused on pain and more basic function/mobility as individual outcomes demonstrating that TJR is a wellestablished, effective intervention for people with moderate to severe hip or knee osteoarthritis resulting in large and significant improvements in pain and functional limitations (Chang et al., 1996; Fortin et al., 1999; W. H. Harris \& Sledge, 1990; Judge et al., 2010; Laupacis et al., 1993). This is despite literature that indicates that return to higher demand activities and participation in 
social roles, leisure pursuits and community interactions are important to people undergoing TJR (Gignac et al., 2008; Rastogi et al., 2007). Few studies have evaluated recovery at multiple time points or they have used a limited range of outcomes or measures that combine physical function and social and leisure activities as one construct (Bachmeier et al., 2001; Kennedy et al., 2008; Zimmerman et al., 2002). Only the study by Pollard et al. simultaneously evaluated the relationship of the ICF components in people prior to TJR (Pollard et al., 2010). These authors found significant relationships between impairment and activity limitations and between activity limitation and participation restrictions using the Aberdeen suite of measures. The relationship between impairment and participation restrictions was not significant. This latter finding is in contrast to our work that found a significant relationship between physical impairment and participation restrictions pre-surgery and also at all follow-up times with the exception of the two week post surgical follow-up. The difference in these findings is unknown, but it may stem from the different measures used in the two studies.

A critical finding from our work was that when people were recovering, the magnitude of the cross-sectional relationships varied over time. Although the purpose of the work by Harris et al was to evaluate if the ICF components predicted health, their work also demonstrated that the correlations between components varied from 0.46 to 0.79 during recovery from distal radius fracture (J. E. Harris et al., 2005). There also was variability in the magnitude of the correlations of impairment to participation restriction and activity limitation to participation restriction but the pattern was variable in that the correlations increased from time 1 to time 2 and then decreased to time three. These findings are similar to our work conducted with path analysis where the magnitude of the path coefficients did not suggest a linear relationship at sequential time points. Understanding this pattern of relationships was beyond the scope of the current work 
but we speculate that as people recover personal and environmental factors may be influential and that these may differentially affect the relationships over time.

This work has focused on the relationships of the ICF but the interpretation of the findings also needs to be supported by clinical context. Our findings are plausible as they overall are in keeping with the expected clinical recovery of people with TJR. As expected, physical impairments improved sooner than activity limitations, although both improved early in the post operative period, whereas participation restrictions only started to show improvement at 3 months post surgery. Additionally, while our a priori hypothesized model identified most of the relationships, we also needed to adjust our analyses for covariates that have been shown in other work to influence outcome in people with joint replacement to achieve a well-fitting model. These covariates included age, sex, hip versus knee replacement, BMI, low back pain and mood (Bachmeier et al., 2001; Bourne et al., 2007; Jones et al., 2007; Jones et al., 2000; Krushell \& Fingeroth, 2007; Naylor et al., 2008). So, while not in our initial model, adjustment by these covariates is also plausible.

We used a single framework, the ICF, to guide our choice of outcome measures. There are a number of other disability frameworks (Masala \& Petretto, 2008) and we cannot be sure that they would similarly demonstrate these variable relationships among (between) concepts over time. However, there is similarity in many of the concepts of these disability models such that we expect that there would be similarity in the relationships (Badley, 2008).

In choosing our measures to represent the ICF components, we selected those that are commonly reported for TJR (Riddle et al., 2008). We deliberately chose measures that represented activity limitations and participation restrictions as separate entities. Although not the intent of this work, our results support the opinion (Badley, 2008; A. M. Jette et al., 2003; 
Nordenfelt, 2003; Schuntermann, 2005; Simeonsson et al., 2003) and work of others (A. M. Jette et al., 2007; Pollard et al., 2010) suggesting that there is a distinction between activity and participation. It must, however, also be recognized that there continues to be debate on the definition of participation and how it should be measured (Dijkers, 2010). Our work has used but one measure with one approach to conceptualization and measurement.

We constructed measured variables to represent the ICF components using standardized measures based on those commonly used for clinical purposes as we wanted to ensure this theoretically-based work was grounded in the real clinical world. As a result, we did not have a sufficient number of variables to construct latent variables for all ICF components. Hence, we evaluated the internal consistency of the measured variables representing the ICF constructs to support our decision to combine these observed variables. For all but activity limitations, the Cronbach's alpha was very close to or above 0.80 supporting the internal consistency of the measured variable. The activity limitations component score was constructed based on two standardized measures and alpha was closer to 0.70 . While the small number of measures likely contributed to this lower than desired value, the results must be interpreted considering the potential impact of this lower level of precision. Additionally, we would recommend that future work evaluating the ICF component relationships include sufficient measures to allow for the construction of latent variables for each ICF construct. This would allow the testing of and inclusion of the measurement model within a structural equation model analysis approach.

The results of this work are from a sample that is improving a great deal and rapidly. Further study is required to determine if variability in the relationships of the ICF components occurs in the face of deterioration, smaller changes, or slower courses of recovery. Additionally, we have not considered the impact of personal and environmental contextual factors on the ICF 
components. We adjusted for a limited number of personal factors but we would see our work as a first step in understanding the complexity of the relationships of the ICF components.

The findings of this study provide additional and significant information about the longitudinal relationships of the components of the ICF framework, particularly in its application in the context of intervention and recovery. Evaluating the effect of contextual factors is important future work. 


\section{References}

Allison, P. D. (2003). Missing data techniques for structural equation modeling. Journal of Abnormal Psychology, 112(4), 545-557.

Bachmeier, C. J., March, L. M., Cross, M. J., Lapsley, H. M., Tribe, K. L., Courtenay, B. G., et al. (2001). A comparison of outcomes in osteoarthritis patients undergoing total hip and knee replacement surgery. Osteoarthritis and Cartilage, 9(2), 137-146.

Badley, E. M. (2008). Enhancing the conceptual clarity of the activity and participation components of the International Classification of Functioning, Disability, and Health. Social Science \& Medicine, 66(11), 2335-2345.

Badley, E. M., \& Tennant, A. (1993). Impact of disablement due to rheumatic disorders in a British population: estimates of severity and prevalence from the Calderdale Rheumatic Disablement Survey. Annals of the Rheumatic Diseases, 52(1), 6-13.

Bellamy, N., Buchanan, W. W., Goldsmith, C. H., Campbell, J., \& Stitt, L. W. (1988). Validation study of WOMAC: a health status instrument for measuring clinically important patient relevant outcomes following total hip or knee arthroplasty in osteoarthritis. Journal of Orthopaedic Rheumatology, 1, 95-108.

Bollen, K. A., \& Long, J. S. (1993). " Introduction". In Testing Structural Equation Models, edited by K.A. Bollen \& J.S. Long. Newsbury Park, CA: Sage Publications;1993, pp.1-9.

Bourne, R., Mukhi, S., Zhu, N., Keresteci, M., \& Marin, M. (2007). Role of obesity on the risk for total hip or knee arthroplasty. Clinical Orthopaedics \& Related Research, 465, 185188. 
Browne, M. W., \& Cuceck, R. (1993). "Alternative ways of assessing model fit" in Testing Structural Equation Models, edited by K.A. Bollen \& J.S. Long Newsbury Park, CA: Sage Publications;1993, pp.136-162.

Canadian Institutes of Health Information. (June 2001.). National Rehabilitation System.

Chang, R. W., Pellisier, J. M., \& Hazen, G. B. (1996). A cost-effectiveness analysis of total hip arthroplasty for osteoarthritis of the hip. Journal of the American Medical Association, 275(11), 858-865.

Dagfinrud, H., Kjeken, I., Mowinckel, P., Hagen, K. B., \& Kvien, T. K. (2005). Impact of functional impairment in ankylosing spondylitis: impairment, activity limitation, and participation restrictions. Journal of Rheumatology, 32(3), 516-523.

Davis, A. M., Badley, E. M., Beaton, D. E., Kopec, J., Wright, J. G., Young, N. L., et al. (2003). Rasch analysis of the Western Ontario McMaster (WOMAC) Osteoarthritis Index: results from community and arthroplasty samples. J Clin Epidemiol, 56(11), 1076-1083.

Dekker, M., Hoeksma, A. F., Dekker, J. H., van Rossum, M. A., Dolman, K. M., Beckerman, H., et al. (2010). Strong relationships between disease activity, foot-related impairments, activity limitations and participation restrictions in children with juvenile idiopathic arthritis. Clinical \& Experimental Rheumatology, 28(6), 905-911.

Dijkers, M. P. (2010). Issues in the conceptualization and measurement of participation: an overview. Archives of Physical Medicine \& Rehabilitation, 91(9 Suppl), S5-16.

Enders, C. K. (2010). Applied missing data analysis. New York: The Guildford Press.

Finney, S. J., \& DiStefano, C. (2006). Non-normal and categorical data in structural equation modeling. Greenwich, CT: Information Age Publishing. 
Fortin, P. R., Clarke, A. E., Joseph, L., Liang, M. H., Tanzer, M., Ferland, D., et al. (1999). Outcomes of total hip and knee replacement: preoperative functional status predicts outcomes at six months after surgery. Arthritis \& Rheumatism, 42(8), 1722-1728.

Fortin, P. R., Penrod, J. R., Clarke, A. E., St-Pierre, Y., Joseph, L., Belisle, P., et al. (2002). Timing of total joint replacement affects clinical outcomes among patients with osteoarthritis of the hip or knee. Arthritis \& Rheumatism, 46(12), 3327-3330.

Gignac, M. A., Backman, C. L., Davis, A. M., Lacaille, D., Mattison, C. A., Montie, P., et al. (2008). Understanding social role participation: what matters to people with arthritis? Journal of Rheumatology, 35(8), 1655-1636.

Hamilton, B., Granger, C., \& Sherwin, F. (1987). A uniform national data system for medical rehabilitation. In Fuhrer J, ed. Rehabilitation Outcomes: Analysis Measurement. Baltimore, MD: Paul H. Brookes; 1987, pp.137-147.

Harris, J. E., MacDermid, J. C., \& Roth, J. (2005). The International Classification of Functioning as an explanatory model of health after distal radius fracture: a cohort study. Health \& Quality of Life Outcomes, 3, 73.

Harris, W. H., \& Sledge, C. B. (1990). Total hip and total knee replacement (2). New England Journal of Medicine 323(12), 801-807.

Herrmann, C. (1997). International experiences with the Hospital Anxiety and Depression Scale-a review of validation data and clinical results. Journal of Psychosomatic Research, 42(1), 17-41.

Hu, L. T., \& Bentler, P. M. (1998). Fit indices in covariance structure modeling: sensitivity to underparameterized model misspecification. Psychological Methods, 3, 424-453. 
Institute for Clinical Evaluative Sciences. (2000). Urgency Rating, Waiting List Management and Patient Outcomes Monitoring for Primary Hip/Knee Joint Replacement. pp. 1-73). Toronto, Canada; 2000, pp. 1-73.

Jette, A. M., Haley, S. M., Coster, W. J., Kooyoomjian, J. T., Levenson, S., Heeren, T., et al. (2002). Late life function and disability instrument: I. Development and evaluation of the disability component. J Gerontol A Biol Sci Med Sci, 57(4), M209-216.

Jette, A. M., Haley, S. M., \& Kooyoomjian, J. M. (2006). Late Life FDI Manual. Sargent College of Health and Rehabilitation Sciences, Boston University: Boston University: Roybal Center for Enhancement of Late-Life Function.

Jette, A. M., Haley, S. M., \& Kooyoomjian, J. T. (2003). Are the ICF Activity and Participation dimensions distinct? Journal of Rehabilitation Medicine, 35(3), 145-149.

Jette, A. M., Tao, W., \& Haley, S. M. (2007). Blending activity and participation sub-domains of the ICF. Disability \& Rehabilitation, 29(22), 1742-1750.

Jones, C. A., Beaupre, L. A., Johnston, D. W., \& Suarez-Almazor, M. E. (2007). Total joint arthroplasties: current concepts of patient outcomes after surgery. Rheumatic Diseases Clinics of North America, 33(1), 71-86.

Jones, C. A., Voaklander, D. C., Johnston, D. W., \& Suarez-Almazor, M. E. (2000). Health related quality of life outcomes after total hip and knee arthroplasties in a community based population. Journal of Rheumatology 27(7), 1745-1752.

Judge, A., Cooper, C., Williams, S., Dreinhoefer, K., \& Dieppe, P. (2010). Patient-reported outcomes one year after primary hip replacement in a European Collaborative Cohort. Arthritis Care \& Research (Hoboken), 62(4), 480-488. 
Kennedy, D. M., Stratford, P. W., Riddle, D. L., Hanna, S. E., \& Gollish, J. D. (2008). Assessing recovery and establishing prognosis following total knee arthroplasty. Physical Therapy, $88(1), 22-32$.

Kline, R. B. (2005). Principles and Practice of Structural Equation Modeling. New York: Guilford Press.

Krushell, R. J., \& Fingeroth, R. J. (2007). Primary Total Knee Arthroplasty in Morbidly Obese Patients: a 5- to 14-year follow-up study. Journal of Arthroplasty, 22(6 Suppl 2), 77-80.

Lai, J. S., Crane, P. K., \& Cella, D. (2006). Factor analysis techniques for assessing sufficient unidimensionality of cancer related fatigue. Qual Life Res, 15(7), 1179-1190.

Laupacis, A., Bourne, R., Rorabeck, C., Feeny, D., Wong, C., Tugwell, P., et al. (1993). The effect of elective total hip replacement on health-related quality of life. Journal of Bone \& Joint Surgery, 75(11), 1619-1626.

Little, T. D., Cunningham, W. A., Shahar, G., \& Widaman, K. (2002). To Parcel or Not to Parcel: Exploring the Question, Weighing the Merits', Structural Equation Modeling. A Multidisciplinary Journal, 9(2), 151-173.

MacCallum, R. C., Roznowski, M., \& Necowitz, L. B. (1992). Model modifications in covariance structure analysis: the problem of capitalization on chance. Psychol Bull, 111(3), 490-504.

Machado, G. P., Gignac, M. A., \& Badley, E. M. (2008). Participation restrictions among older adults with osteoarthritis: a mediated model of physical symptoms, activity limitations, and depression. Arthritis \& Rheumatism, 59(1), 129-135.

Masala, C., \& Petretto, D. R. (2008). From disablement to enablement: conceptual models of disability in the 20th century. Disability \& Rehabilitation, 30(17), 1233-1244. 
Muthen, L. K., \& Muthen, B. O. (1998-2009). Mplus: Statistical Analysis with Latent Variables User's Guide. Los Angeles, CA,.

Naylor, J. M., Harmer, A. R., \& Heard, R. C. (2008). Severe other joint disease and obesity independently influence recovery after joint replacement surgery: an observational study. Australian Journal of Physiotherapy, 54(1), 57-64.

Nilsdotter, A. K., Lohmander, L. S., Klassbo, M., \& Roos, E. M. (2003). Hip disability and osteoarthritis outcome score (HOOS)--validity and responsiveness in total hip replacement. BMC Musculoskelet Disord, 4, 10.

Nordenfelt, L. (2003). Action theory, disability and ICF. Disability \& Rehabilitation, 25(18), 1075-1079.

O'Connor, P. J. (2004). Evaluation of four highly cited energy and fatigue mood measures. Journal of Psychosomatic Research, 57(5), 435-441.

Pisoni, C., Giardini, A., Majani, G., \& Maini, M. (2008). International Classification of Functioning, Disability and Health (ICF) core sets for osteoarthritis. A useful tool in the follow-up of patients after joint arthroplasty. European Journal of Physical \& Rehabilitation Medicine, 44(4), 377-385.

Pollard, B., Johnston, M., \& Dieppe, P. (2010). Exploring the relationships between Inrternational Classification of Functioning, Disability and Health (ICF) constructs of impairment, joint replacement. BMC Musculoskelet Disord, 12(1), 97.

Rastogi, R., Davis, A. M., \& Chesworth, B. M. (2007). A cross-sectional look at patient concerns in the first six weeks following primary total knee arthroplasty. Health \& Quality of Life Outcomes, 5, 48. 
Riddle, D. L., Stratford, P. W., \& Bowman, D. H. (2008). Findings of extensive variation in the types of outcome measures used in hip and knee replacement clinical trials: a systematic review. Arthritis \& Rheumatism, 59(6), 876-883.

Roos, E. M., Roos, H. P., Lohmander, L. S., Ekdahl, C., \& Beynnon, B. D. (1998). Knee Injury and Osteoarthritis Outcome Score (KOOS)--development of a self-administered outcome measure. Journal of Orthopaedic \& Sports Physical Therapy, 28(2), 88-96.

Rusch, M., Nixon, S., Schilder, A., Braitstein, P., Chan, K., \& Hogg, R. S. (2004). Impairments, activity limitations and participation restrictions: prevalence and associations among persons living with HIV/AIDS in British Columbia. Health \& Quality of Life Outcomes, $2,46$.

Ryser, L., Wright, B. D., Aeschlimann, A., Mariacher-Gehler, S., \& Stucki, G. (1999). A new look at the Western Ontario and McMaster Universities Osteoarthritis Index using Rasch analysis. Arthritis Care Res, 12(5), 331-335.

Sangha, O., Stucki, G., Liang, M. H., Fossel, A. H., \& Katz, J. N. (2003). The Self-Administered Comorbidity Questionnaire: a new method to assess comorbidity for clinical and health services research. Arthritis \& Rheumatism, 49(2), 156-163.

SAS Institute Inc. (2005). SAS/STAT User's Guide. Version 9. SAS Institute Inc. Cary, NC.

Schuntermann, M. F. (2005). The implementation of the International Classification of Functioning, Disability and Health in Germany: experiences and problems. International Journal of Rehabilitation Research, 28(2), 93-102.

Schwarzkopf, S. R., Ewert, T., Dreinhofer, K. E., Cieza, A., \& Stucki, G. (2008). Towards an ICF Core Set for chronic musculoskeletal conditions: commonalities across ICF Core 
Sets for osteoarthritis, rheumatoid arthritis, osteoporosis, low back pain and chronic widespread pain. Journal of Clinical Rheumatology, 27(11), 1355-1361.

Simeonsson, R. J., Leonardi, M., Lollar, D., Bjorck-Akesson, E., Hollenweger, J., \& Martinuzzi, A. (2003). Applying the International Classification of Functioning, Disability and Health (ICF) to measure childhood disability. Disability \& Rehabilitation, 25(11-12), 602-610.

Slim, F. J., van Schie, C. H., Keukenkamp, R., Faber, W. R., \& Nollet, F. (2010). Effects of impairments on activities and participation in people affected by leprosy in The Netherlands. Journal of Rehabilitation Medicine, 42(6), 536-543.

Ustun, T. B., Chatterji, S., Bickenbach, J., Kostanjsek, N., \& Schneider, M. (2003). The International Classification of Functioning, Disability and Health: a new tool for understanding disability and health. Disability \& Rehabilitation, 25(11-12), 565-571.

Von Korff, M., Jensen, M. P., \& Karoly, P. (2000). Assessing global pain severity by self-report in clinical and health services research. Spine, 25(24), 3140-3151.

Von Korff, M., Ormel, J., Keefe, F. J., \& Dworkin, S. F. (1992). Grading the severity of chronic pain. Pain, 50(2), 133-149.

World Health Organization (2001). WHO International Classification of Functioning, Disability and Health, Geneva

World Health Organization (2001, Page 12). WHO International Classification of Functioning, Disability and Health, Geneva.

World Health Organization (2001, Page 14). WHO International Classification of Functioning, Disability and Health, Geneva.

World Health Organization (2001, Page 18). WHO International Classification of Functioning, Disability and Health, Geneva. 
Xie, F., Lo, N. N., Lee, H. P., Cieza, A., \& Li, S. C. (2008). Validation of the International Classification of Functioning, Disability, and Health (ICF) Brief Core Set for osteoarthritis. Scand J Rheumatol, 37(6), 450-461.

Zigmond, A. S., \& Snaith, R. P. (1983). The hospital anxiety and depression scale. Acta Psychiatrica Scandinavica, 67(6), 361-370.

Zimmerman, S., Hawkes, W. G., Hudson, J. I., Magaziner, J., Hebel, J. R., Towheed, T., et al. (2002). Outcomes of surgical management of total HIP replacement in patients aged 65 years and older: cemented versus cementless femoral components and lateral or anterolateral versus posterior anatomical approach. Journal of Orthopaedic Research, 20(2), 182-191. 
Table 1: ICF component scores over time in people with primary hip or knee replacement ( $\mathbf{n}=931)$

\begin{tabular}{|c|c|c|c|c|c|c|}
\hline Measure & $\begin{array}{c}\text { Pre-surgery } \\
\text { mean (sd) }\end{array}$ & $\begin{array}{c}2 \text { weeks } \\
\text { mean (sd) }\end{array}$ & $\begin{array}{l}1 \text { month } \\
\text { mean }(\mathrm{sd})\end{array}$ & $\begin{array}{l}3 \text { months } \\
\text { mean (sd) }\end{array}$ & $\begin{array}{l}6 \text { months } \\
\text { mean (sd) }\end{array}$ & $\begin{array}{l}12 \text { months } \\
\text { mean (sd) }\end{array}$ \\
\hline $\begin{array}{l}\text { Physical } \\
\text { Impairment } \\
\text { (possible score } \\
\text { range 0-20) }\end{array}$ & $\begin{array}{l}11.27 \\
(3.79)\end{array}$ & $\begin{array}{c}7.63 \\
(4.07)\end{array}$ & $\begin{array}{c}5.89 \\
(3.80)\end{array}$ & $\begin{array}{c}3.86 \\
(3.37)\end{array}$ & $\begin{array}{c}3.46 \\
(3.50)\end{array}$ & $\begin{array}{c}2.84 \\
(3.17)\end{array}$ \\
\hline $\begin{array}{l}\text { Activity Limitations } \\
\text { (possible score } \\
\text { range 0-20) }\end{array}$ & $\begin{array}{l}14.06 \\
(2.89)\end{array}$ & $\begin{array}{l}13.52 \\
(2.61)\end{array}$ & $\begin{array}{l}11.78 \\
(2.99)\end{array}$ & $\begin{array}{c}9.06 \\
(3.79)\end{array}$ & $\begin{array}{c}8.21 \\
(4.18)\end{array}$ & $\begin{array}{c}7.56 \\
(4.21)\end{array}$ \\
\hline $\begin{array}{l}\text { Participation } \\
\text { Restrictions } \\
\text { (possible score } \\
\text { range 0-30) } \\
\end{array}$ & $\begin{array}{l}12.93 \\
(5.02)\end{array}$ & $\begin{array}{l}17.62 \\
(5.48)\end{array}$ & $\begin{array}{l}13.68 \\
(5.65)\end{array}$ & $\begin{array}{c}7.83 \\
(5.11)\end{array}$ & $\begin{array}{c}6.84 \\
(4.93)\end{array}$ & $\begin{array}{c}6.38 \\
(4.76)\end{array}$ \\
\hline
\end{tabular}

Note: For component scores, zero represents no impairment, no activity limitation and no participation restrictions. Each component score was derived by standardized measure scores converted to 0 to 10 and then summing the measure scores. The measures were the KOOS/HOOS-pain subscale and Chronic Pain Grade for Physical Impairment; WOMAC function subscale and HOOS/KOOS function in Sports and Recreation subscale; and, the Late Life Disability Index frequency and limitations subscales and the community mobility measure adapted from the Calderdale Rheumatic Disablement Survey. 
Table 2: Unstandardized coefficients for the final model pathways when direct paths between ICF components over subsequent times were constrained to be equal

\begin{tabular}{|c|c|c|c|}
\hline \multicolumn{4}{|c|}{ Physical Impairment $\rightarrow$ Activity Limitations } \\
\hline Time & Total & Direct & Indirect \\
\hline pre-surgery to 2 weeks & .084 & -.212 & .295 \\
\hline 2 weeks to 1 month & 394 & -.212 & .606 \\
\hline 1 to 3 months & .408 & -.212 & .620 \\
\hline 3 to 6 months & .566 & -.212 & .778 \\
\hline 6 to 12 months & .359 & -.212 & .571 \\
\hline \multicolumn{4}{|c|}{ Activity Limitations $\rightarrow$ Participation Restrictions } \\
\hline Time & Total & Direct & Indirect \\
\hline pre-surgery to 2 weeks & .433 & -.297 & .730 \\
\hline 2 weeks to 1 month & 1.052 & -.297 & 1.349 \\
\hline 1 to 3 months & .383 & -.297 & .680 \\
\hline 3 to 6 months & .382 & -.297 & .679 \\
\hline 6 to 12 months & 247 & -.297 & .544 \\
\hline \multicolumn{4}{|c|}{ Physical Impairment $\rightarrow$ Participation Restrictions } \\
\hline Time & Total & Direct & Indirect \\
\hline pre-surgery to 2 weeks & 103 & 0 & .103 \\
\hline 2 weeks to 1 month & .593 & 0 & .593 \\
\hline 1 to 3 months & .452 & 0 & .452 \\
\hline 3 to 6 months & .628 & 0 & .628 \\
\hline 6 to 12 months & .435 & 0 & .435 \\
\hline
\end{tabular}

Notes: All times are post surgery except where specified as pre-surgery. Model adjusted for age, sex, knee versus hip replacement, body mass index, low back pain and mood. All coefficients were statistically significant $p<0.05$. The results show the unstandardized total, direct and indirect pathway coefficients when the pathways between components at sequential times (i.e. physical impairment pre-surgery to activity limitations at 2 weeks post surgery etc and activity limitations pre-surgery to participation restrictions at 2 weeks post surgery) were constrained to be equal. This model was parsimonious in that it was not statistically significantly different from the base model. 


\section{Figure Captions}

Figure 1: Hypothesized model showing interrelationships of the ICF components over time

Figure 2: Final model: Relationships based on fully standardized coefficients among impairments, activity limitations and participation restrictions over time adjusting for covariates: age, sex, hip versus knee replacement, obesity, low back pain and mood. Model Fit Indices: $\mathrm{CFI}=0.949 ; \mathrm{TLI}=0.932 ; \mathrm{RMSEA} 0.057 ; \mathrm{SRMR} 0.084$

\section{Acknowledgement}

The ICF Research Team includes in alphabetical order:

EM Badley, Division of Health Care and Outcomes Research, Toronto Western Research Institute and Dalla Lana School of Public Health, University of Toronto, Toronto, Canada

DE Beaton, Keenan Research Institute, St. Michael's and Departments of Occupational Therapy and Rehabilitation Science, University of Toronto, Toronto, Canada

P Côté, Division of Health Care and Outcomes Research, Toronto Western Research Institute and Dalla Lana School of Public Health, University of Toronto, Toronto, Canada

AM Davis, Division of Health Care and Outcomes Research, Toronto Western Research Institute; Departments of Rehabilitation Science and Health Policy, Management and Evaluation, University of Toronto, Toronto, Canada

J Flannery, Department of Physiatry, University of Toronto and Toronto Rehabilitation Institute, Toronto, Canada 
MA Gignac, Division of Health Care and Outcomes Research, Toronto Western Research Institute and Dalla Lana School of Public Health, University of Toronto, Toronto, Canada

S Hogg-Johnson, Institute for Work and Health, Toronto, Canada

S Ibrahim, Institute for Work and Health, Toronto, Canada

N Mahomed, Division of Orthopaedic Surgery, University of Toronto and The Arthritis

Program, Toronto Western Hospital, Toronto, Canada

AV Perruccio, Department of Orthopaedic Surgery, Toronto Western Hospital and Institute of Health Policy, Management and Evaluation, University of Toronto Toronto, Canada

E Schemitsch, Division of Orthopaedic Surgery, University of Toronto and St. Michael's, Toronto, Canada

D Streiner, Departments of Psychiatry, University of Toronto, Toronto, Canada and McMaster University, Hamilton, Canada

R Wong, Division of Health Care and Outcomes Research and Arthritis Community Research and Evaluation Unit, Toronto Western Research Institute, Toronto, Canada

This work was supported by an operating grant from the Canadian Institutes of Health Research, grant number 77518 . 
Figure 1

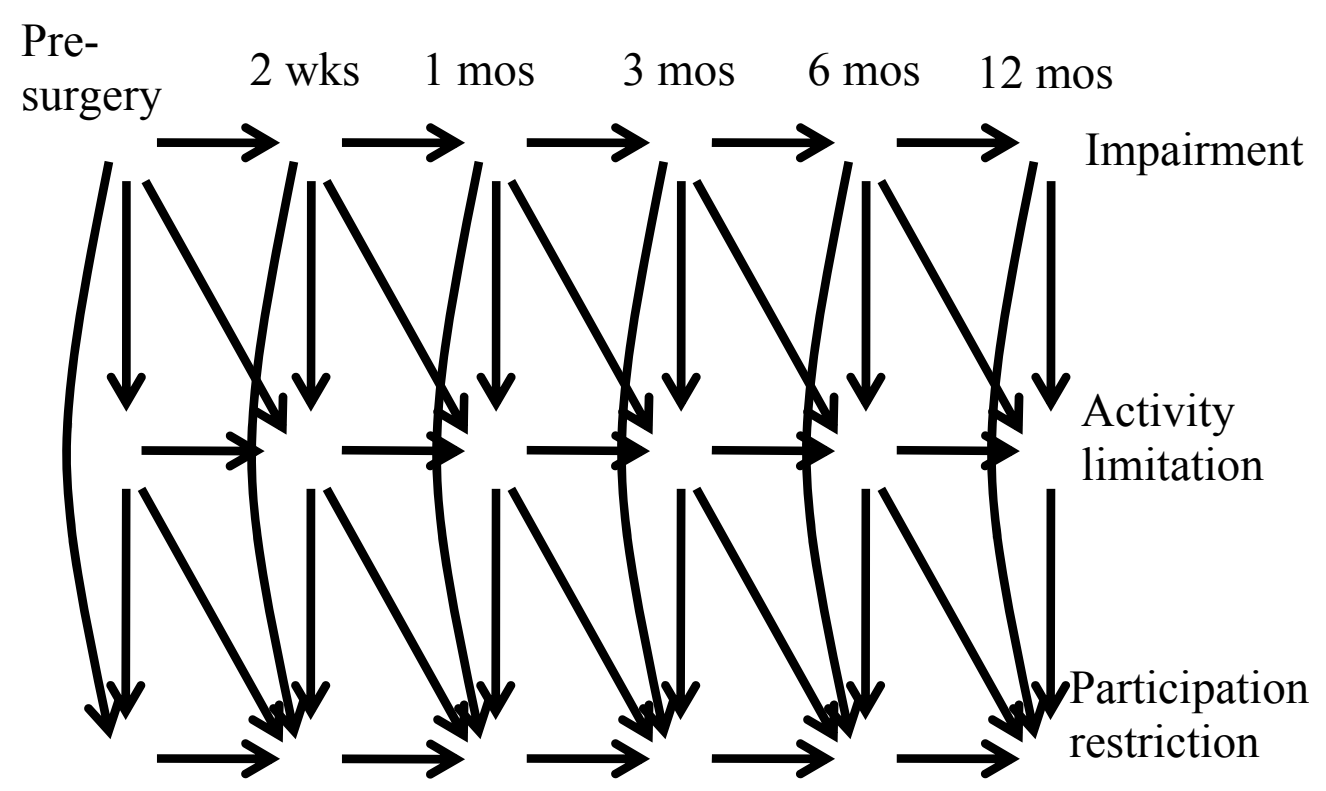


Figure 2

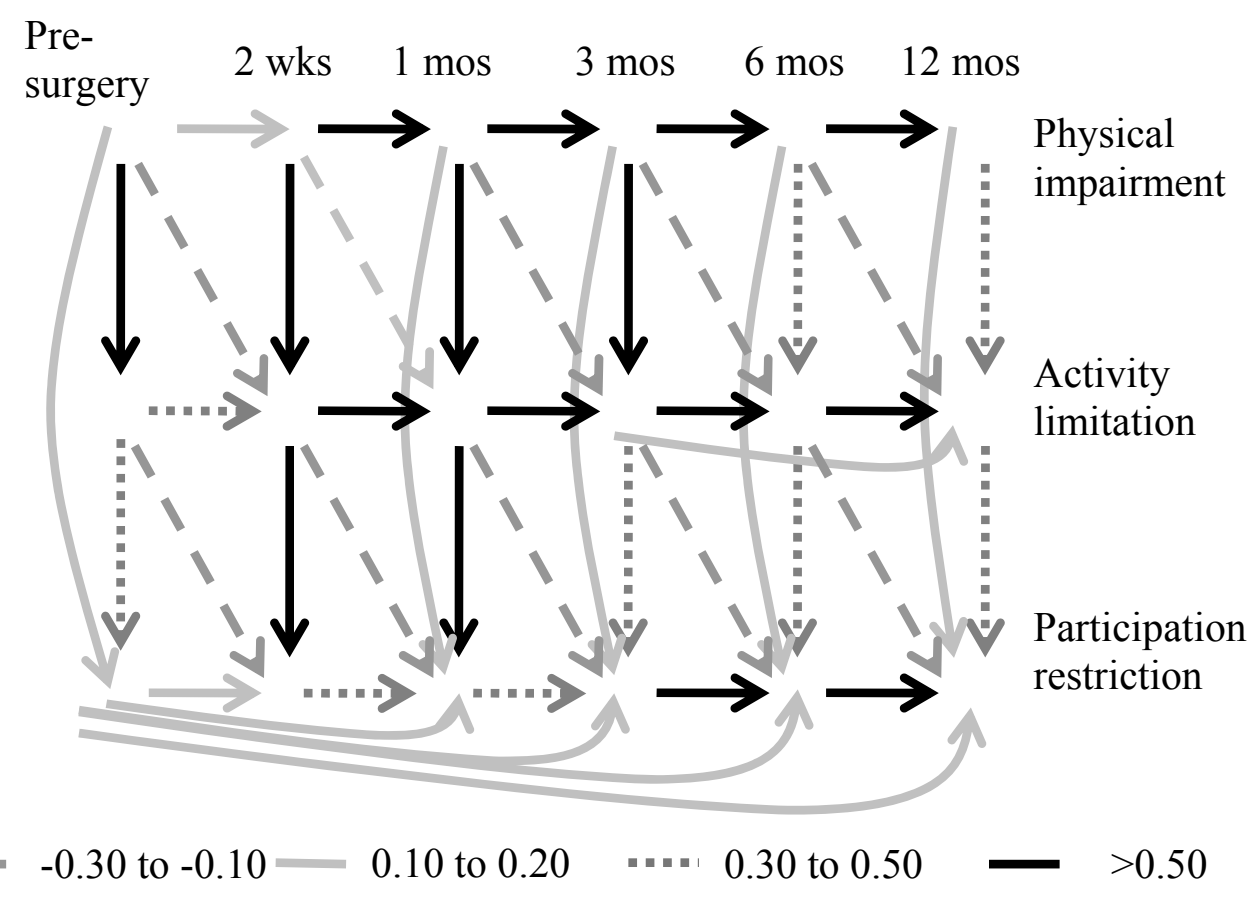

Coefficients: $-\quad-0.30$ to $-0.10=0.10$ to $0.20 \quad 0.30$ to $0.50 \longrightarrow 0.50$ 